Methoden

Egidio Leo* and Sebastian Engell

\title{
Integrated day-ahead energy procurement and production scheduling
}

\author{
Integrierte day-ahead-Energiebeschaffung und Produktionsplanung
}

https://doi.org/10.1515/auto-2018-0016

Received February 18, 2018; accepted May 7, 2018

Abstract: For the optimal operation of power-intensive plants, a challenge which is addressed in this work is to simultaneously determine the optimal production schedule and the optimal day-ahead electricity commitment. In order to ensure stability of the power grid, the electricity suppliers impose a daily electricity commitment to large consumers. The consumers have to commit one day in advance to the amount of energy they are going to purchase and use for a horizon of 24 hours (with an hourly discretization) and in case the actual electricity consumption differs significantly from the committed profile, the consumer is obliged to pay penalties. Since the consumers have to commit to the electricity suppliers before the actual electricity demand is known, uncertainty needs to be taken into account. A stochastic mixed-integer linear programming model is developed to consider two critical sources of uncertainty: equipment breakdowns and deviation prices. Equipment breakdowns can reduce the production capacity and make the actual electricity consumption deviate from the day-ahead electricity commitment. The application of the proposed approach to a continuous power-intensive plant shows the benefit gained from the solution of the stochastic model instead of the deterministic counterpart in terms of reduction of the cost of the energy.

Keywords: production scheduling, stochastic programming, demand side management, load deviations

Zusammenfassung: Dieser Beitrag behandelt die simultane Produktionsplanung und day-aheadStrombeschaffung für energieintensive Produktionspro-

\footnotetext{
*Corresponding author: Egidio Leo, Process Dynamics and Operations Group, Department of Biochemical and Chemical Engineering, Technische Universität Dortmund, Emil-Figge-Str.70, 44221 Dortmund, Germany, e-mail: egidio.leo@tu-dortmund.de Sebastian Engell, Process Dynamics and Operations Group, Department of Biochemical and Chemical Engineering, Technische Universität Dortmund, Emil-Figge-Str.70, 44221 Dortmund, Germany, e-mail: sebastian.engell@tu-dortmund.de
}

zesse. Um die Stabilität des Stromnetzes zu gewährleisten, fragen die Versorger bei großen Verbrauchern einen Tag im voraus den stündlichen Energiebezug ab und wenn sich der geplante Verlauf und der reale Verbrauch unterscheiden, fallen für den Verbraucher Zusatzkosten an. Bei der Planung müssen Unsicherheiten über den tatsächlichen Produktionsablauf und die Höhe der Zusatzkosten berücksichtige werden. Es wird eine gemischt-ganzzahlige Problemformulierung vorgestellt, bei der Anlagenausfälle und variierende Zusatzkosten berücksichtigt werden. Die Anwendung des vorgeschlagenen Modells auf ein Beispiel zeigt, dass sich erhebliche Vorteile aus der Lösung des stochastischen Modells anstelle einer deterministischen Formulierung ergeben.

Schlagwörter: Produktionsplanung, stochastische Optimierung, Energie-Abweichungen

\section{Introduction}

Industrial Demand-Side Management (iDSM) has been recognized as an important means to support the integration of renewable energy sources into the electricity grid ([23]). The official target of the energy sector in Germany for the year 2020, as well in other European countries, is to provide more than $30 \%$ of electric power from renewables ([8]). The difficulties of relying on renewable energy sources are related to the volatile power feed due to the nature of the sources, e. g., wind energy, that are not linked to the power needs. This effects drastically the electricity price in the spot market and generates supply-demand mismatches compromising the stability of the power grid. iDSM is expected to play a crucial role to operate the power grid in a more efficient way and to cope with the possible drawbacks of the integration of renewable energy sources. At the same time, iDSM can create additional profit for the electricity consumers $([20,28,32])$. In fact, iDSM can be viewed from two distinct perspectives: the grid operator's perspective and the electricity consumer's perspective. The main objective of the grid operator is to increase 
efficiency and ensure stability of the power grid, reducing electricity demand peaks and quickly reacting to supplydemand mismatches in the grid. To this aim, the grid operator defines iDSM programs with financial incentives. On the other hand, the main objective of the electricity consumer is cost reduction. Therefore, IDSM is a means to exploit the financial incentives set up by the grid operator and to adapt the production levels to dynamic market or supply conditions, e. g., the time-varying electricity prices coming from the spot market. An overview of advances and challenges in the area of iDSM can be found in [32].

In this work, we take the standpoint of large electricity consumers whose main objective is to reduce the energy cost by integrating production scheduling and energy management. For large consumers of electric power, the electricity purchase options include different types of power contracts (Base load, Time-of-Use (TOU)) and different spot markets (day-ahead market, intra-day market, futures market, Over-the-Counter market). Regarding the day-ahead spot market, one key aspect is the hourlyvarying electricity price due to the varying availability of renewable energy sources. Therefore, the capability to adapt the production level to the time-varying price signals coming from the day-ahead spot market is important for the energy-intensive industries to reduce the energy cost. A number of contributions on scheduling models that take into account the time-varying electricity price can be found in the literature $([5,6,21])$. While the volatility of the electricity prices constitutes a potential saving for the electricity consumers, for the grid operator it leads to increased effort to match demand and supply. Therefore, to ensure the stability of the power grid, the grid operator imposes to large consumers a daily day-ahead hourly electricity commitment and in case the actual consumption deviates by more than a small margin from the pre-agreed values, financial penalties which are often in the same range as the net electricity cost are incurred. This is known in literature as the load-deviation problem. In [22], a scheduling solution for electrical load tracking of a steel plant is proposed. The schedule is defined such that the total electricity consumption tracks the load curve as closely as possible while respecting all production constraints. In [13], the authors take into account multiple electricity sources (Base load and Time-Of-Use power contracts, day-ahead market, on-site generation) and the load deviation problem to determine the optimal production schedule.

However, in these contributions the load commitment decisions are assumed as given and are not optimized. In this work, we address the challenge of determining simultaneously the optimal day-ahead electricity commitment and the optimal production schedule. Since decisions regarding the electricity commitment have to be made before the actual electricity demand is known for the time horizon of interest, it is crucial to account for uncertainties. To this end we adopt our recently proposed approach $([18,19])$, where a stochastic programming formulation [3] is applied to model uncertainty about the equipment failures. In this contribution, we extend the results of the proposed approach to a power-intensive plant equipped with a power plant that can be used to generate electricity and therefore to reduce the electricity commitment. Moreover we account for uncertain deviation prices (also called imbalance prices). In fact the deviation prices are determined only after the actual electricity consumption occurs, since only then the electricity grid operator is able to quantify the cost of reacting to a supply-demand mismatch in the power grid. It is important to note that usually there is a correlation between the deviation prices and the dayahead market prices: an over/under-consumption during a demand peak interval is penalized more than during an off-peak interval.

In the stochastic programming approach, the decision process is divided in decision stages and the decision variables are divided into sets that belong to the stages (see Section 2). In the proposed formulation the first-stage variables represent the day-ahead electricity commitment decisions and whether the power plant generates electricity. First-stage decisions have to be made at the beginning of the time horizon and cannot be modified. The second stage decisions react to the realization of the uncertainty. Second-stage variables are the plant operating decisions (production levels, inventories...) and the electricity consumption deviations from the day-ahead commitment.

The remainder of this paper is organized as follows: in Section 2 the two-stage programming formulation is described. In Section 3 the problem statement is presented, before highlighting the uncertainty modeling strategy and the proposed two-stage stochastic MILP in Section 4. The main results are discussed in Section 5 . The results are presented in terms of the Value of the Stochastic Solution (VSS) in Section 6 before drawing some conclusions in Section 7.

\section{Stochastic programming}

A stochastic program is a mathematical program (optimization model) where some of the data is uncertain and can be described by a probability distribution. It is assumed that the random variables have a countable num- 


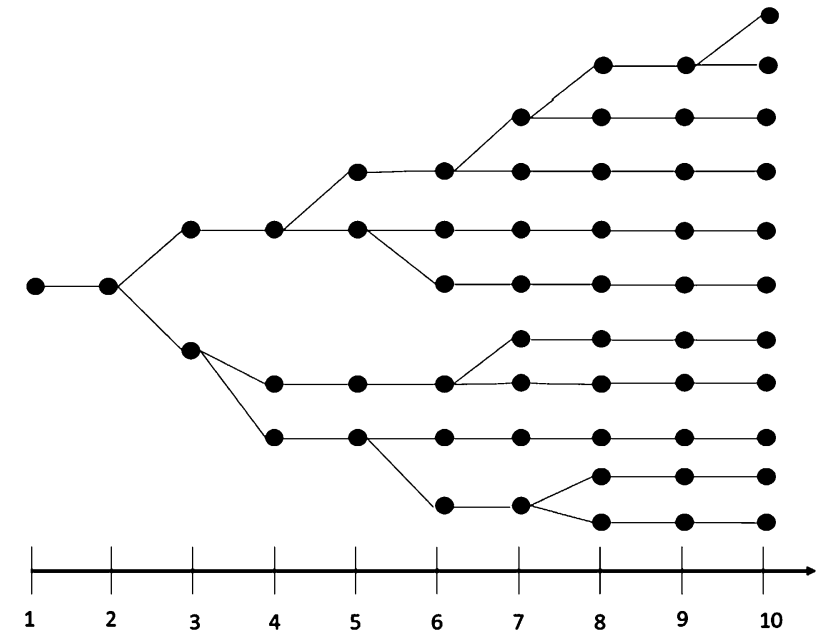

(a)

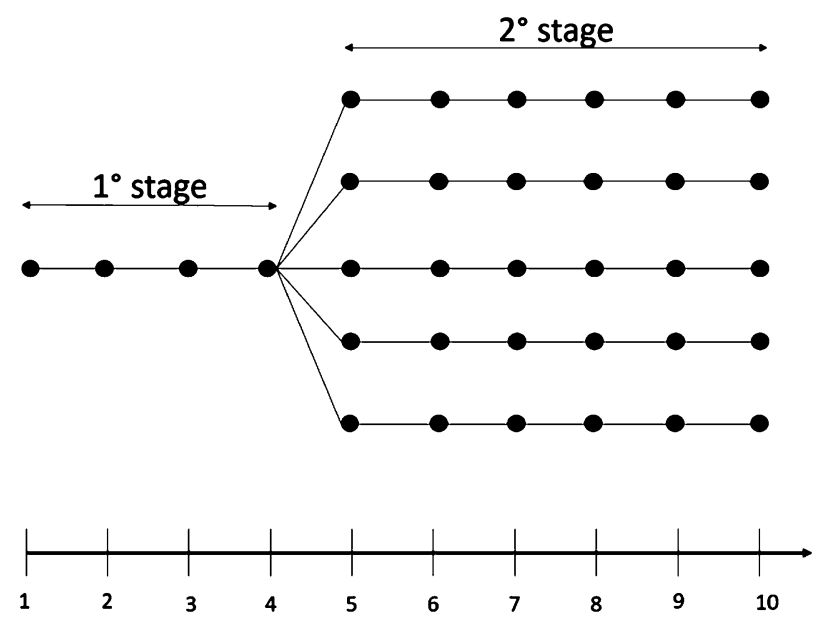

(b)

Figure 1: Multi-stage (a) and two-stage (b) stochastic optimization problems represented by scenario trees.

ber of realizations that are modeled by a discrete set of scenarios $s=1, \ldots, S$. In a stochastic program with recourse, corrective decisions or recourse actions can be taken after the uncertainty has realized. Each point in time at which a decision is made is called a stage. Stochastic programming was introduced by [3] with a two-stage stochastic formulation. In the two-stage stochastic formulation the decision maker takes some actions in the first stage, after which uncertainties affect the outcome of the first-stage decisions. Recourse decisions can then be made in the second stage to adapt to the realization of the uncertainty. A typical example is the planning of a production or distribution facility under uncertainty of the future market, and the later operation according to the real demand. The first stage decisions are optimized under the assumption that recourse decisions will optimally be adapted in the second stage after new information has become available. Thus the total set of $n$ decisions is divided into two groups:

- Decisions that have to be taken before the uncertainty is realized. These are called first-stage or here-and-now decisions and they have to be made at the beginning and cannot be changed over the decision horizon;

- Decisions that have to be taken after the uncertainty has been disclosed. These are called second-stage or wait-and-see decisions and they are a means to react after the realization of the uncertainty.

In [2] the authors extended the two-stage formulation to the more general multi-stage stochastic formulation where the uncertainty is modeled by a scenario tree with $N$ stages that branches at each stage. The decision process progresses along this scenario tree. In stage $i$, the decision is based on the information on the realization of a path in the tree up to this node whereas the future evolution is only known probabilistically. The decisions at stage $i$ are optimized under the assumption that the later decisions are optimally adapted to the information which becomes available after the realization of the uncertainty [9]. Figure 1 shows the scenario-tree representation for multistage (1a) and two-stage (1b) stochastic problems. To solve multi-stage problems, at stage $i$ the reaction of the algorithm to the information obtained at later stages must be taken into account, leading to large-scale optimization problems. A large-scale linear or convex non-linear multistage program with continuous variables can be efficiently solved by applying decomposition algorithms such as Lagrange decomposition ([12]), Progressive hedging ([26]), Benders decomposition also called L-shaped method ([2]) and Generalized Benders decomposition ([11]).

\subsection{Two-stage stochastic integer programming}

When integrality requirements are present, a stochastic program is called Stochastic Mixed-Integer Program (SIP). For linear models and a scenario-based representation of uncertainties, a deterministic equivalent of a two-stage stochastic integer program (2-SSIP) can be stated as the following mixed-integer linear program (MILP) (1):

$$
\begin{array}{ll}
\min _{x, y_{s}} & f\left(x, y_{s}\right)=c^{T} x+\sum_{s=1}^{S} \phi_{s} q_{s}^{T} y_{s} \\
\text { s.t. } & A x \leq b
\end{array}
$$




$$
\begin{aligned}
& T_{s} x+W_{s} y_{s} \leq h_{s} \\
& x \in X, y_{s} \in Y, s=1, \ldots, S
\end{aligned}
$$

The objective of a two-stage stochastic mixed-integer linear program Eq. (1a) consists of the first-stage cost and of the expected value of the second-stage cost. The firststage cost and the second-stage costs are calculated as linear combination of the first-stage variables $x$ and the second-stage variables $y_{s}$ with cost parameters $c$ and $q_{s}$. The expected value of the second-stage costs is calculated by the sum over all the scenarios of the second-stage costs weighted by the corresponding probabilities $\phi_{s}$. The constraints are divided into two groups: the constraints of the first stage Eq. (1b) are related only to the first-stage decisions, the constraints of the second-stage Eq. (1c) include variables of the first-stage and of the second-stage. $A$ and $b$ describe the first-stage parameters and $T_{s}, W_{s}, h_{s}$ represent the parameters of each scenario $s$ of the second-stage. When a solution of the first stage $x$ is always feasible in the second-stage, the stochastic problem has complete recourse. The first-stage variables $x$ and the second-stage variables $y_{s}$ belong to the polyhedral sets $X$ and $Y$. Both the sets $X$ and $Y$ can present integer requirements. In case only the polyhedral set $X$ of the first-stage variables $x$ presents integer requirements, the 2-SSIPs can be efficiently solved applying the decomposition algorithms mentioned above $([17,14])$. This class of problems are called stochastic problems with continuous recourses. When the polyhedral set $Y$ of the second-stage variables $y$ presents integer requirements, the problems are called stochastic problems with integer recourses and the solution becomes harder since decomposition approaches such as Benders decomposition cannot be directly applied. More sophisticated algorithms were developed to derive valid and tight cuts for Benders-like approaches $([27,31])$ or to close the duality gap of Lagrangean relaxation for Lagrange-like algorithms $([4,15])$. Recent advances on the solution of stochastic integer problems can be found in ([16]).

\subsection{Risk-averse optimization}

Considering the expected value of the second-stage cost implies that the realizations of the random parameters have no qualitatively different effect. This however is not always true: even a low probability of large losses may not be acceptable. A remedy is to integrate the concept of risk into the optimization problem. Different risk measures have been presented in the literature: Value-at-Risk (VaR), downside risk, Conditional Value-at-Risk (CVaR) [24]. We adopt here the $\mathrm{CVaR}$, since it is a coherent risk measure (it preserves convexity). Since the definition of CVaR relies on the concept of $\mathrm{VaR}$, it is worth to first introduce the definition of VaR. Let us consider that $X$ is a random variable with the meaning of loss. $V a R_{\alpha}$ is defined as the maximum loss that will not be exceeded at a given confidence level $\alpha$ (with $\alpha \in(0,1)$ ) [29]. An equivalent interpretation of $V a R_{\alpha}$ is the $\alpha$-quantile of the distribution of the random variable $X$. Eq. (2) shows the mathematical definition of $V a R_{\alpha}$

$$
\operatorname{VaR}_{\alpha}(X):=\min \{c: P(X \leq c) \geq \alpha\}
$$

Although VaR is a very popular measure of risk (for instance in financial applications), it has undesirable properties such as non-convexity. CVaR is an alternative measure of risk, with more attractive mathematical properties [30]. CVaR is defined as the expected loss, conditional on the fact that the loss exceeds the VaR at the given confidence level $\alpha$. The mathematical definition of CVaR is given by Eq. (3).

$$
\operatorname{CVaR}_{\alpha}(X):=E\left[X \mid X \geq \operatorname{VAR}_{\alpha}(X)\right]
$$

CVaR is a coherent risk measure and it is more conservative than VaR. Most importantly, CVaR can be expressed by a minimization formula proposed in [25] and shown by Eqs. (4)-(5).

$$
\begin{aligned}
\operatorname{CVaR}_{\alpha}(X) & =\min _{\psi} F_{\alpha}(X, \psi) \\
F_{\alpha}(X, \psi) & =\psi+\frac{1}{1-\alpha} E\left[(X-\psi)^{+}\right]
\end{aligned}
$$

where $E[\cdot]$ is the expectation and $(X-\psi)^{+}=\max \{0 ; X-\psi\}$. $\psi$ takes the value of $V a R_{\alpha}$ when the $C V a R_{\alpha}$ is computed by the above formula. Eqs. (4)-(5) can be formulated as linear constraints in an optimization problem and this provides a computationally efficient way of integrating the CVaR into optimization problems as shown in Section 4.2.8.

\subsection{The value of the stochastic solution}

The value of the stochastic solution (VSS) measures the advantage of using a two-stage stochastic program over using the deterministic counterpart where the stochastic parameters have been replaced by their mean values. In other words, it measures the advantage of accounting for the uncertainty and taking into account the recourse decisions. The VSS and the relative $\overline{V S S}$ are defined by Eqs. (6)-(7).

$$
\begin{aligned}
& V S S=z_{E E V}^{*}-z_{2 S S I P}^{*} \\
& \overline{V S S}=\frac{z_{E E V}^{*}-z_{2 S S I P}^{*}}{z_{E E V}^{*}}
\end{aligned}
$$


where $z_{2 S S I P}^{*}$ is the optimal solution of the multistage stochastic problem and $z_{E E V}^{*}$ is the optimal solution of the stochastic problem with first-stage variables obtained by the optimal solution of the deterministic problem where the stochastic parameters have been replaced by their mean values.

In risk-averse optimization, the objective is to minimize a weighted sum of the expected cost and a measure of risk, e. g., the CVaR. Then the VSS and the relative $\overline{V S S}$ are defined by Eqs. (8)-(9).

$$
\begin{aligned}
& V S S=\eta\left(z_{E E V}^{*}-z_{2 S S I P}^{*}\right)+(1-\eta)\left(C V_{E E V}^{*}-C V_{2 S S I P}^{*}\right) \\
& \overline{V S S}=\frac{\eta\left(z_{E E V}^{*}-z_{2 S S I P}^{*}\right)+(1-\eta)\left(C V_{E E V}^{*}-C V_{2 S S I P}^{*}\right)}{\eta z_{E E V}^{*}+(1-\eta) C V_{E E V}^{*}}
\end{aligned}
$$

where $\eta$ is a scalar coefficient that the decision maker can tune to assign priorities to the expected cost and to the risk measure.

\section{Problem statement}

We consider a continuous power-intensive plant that can produce a given set of products with a known and fixed demand. Inventory capacities are considered and products can be purchased on the market at given costs. The operating cost consists of the electricity cost and the cost of purchasing additional products.

Electricity can be purchased from the day-ahead market at an hourly-varying electricity price or can be produced internally at a constant generation cost and a startup cost. To purchase electricity from the day-ahead market the plant has to commit a day in advance to the amount of electricity that will be used for a period of 24 hours.

The goal is to optimize simultaneously the production scheduling and the electricity commitment. Uncertainty in equipment breakdowns and deviation prices is considered. In the proposed approach, the here-and-now decisions in each time period of the scheduling horizon are:

- the mode of operation of the production plant,

- the mode of operation of the power plant,

- the day-ahead electricity commitment.

The wait-and-see decisions are:

- the plant operating conditions: production rates,

- the amount of product purchased,

- the resulting inventory levels,

- the load deviations from the day-ahead electricity commitment.

\section{The 2-SSIP formulation}

\subsection{Modeling of the uncertainty}

To integrate day-ahead electricity commitment and production scheduling, we propose a two-stage stochastic programming approach [3]. We model two crucial sources of uncertainty: equipment breakdowns and deviation prices. Three different levels of uncertainty - low, medium, and high - are considered for each source of uncertainty. For the equipment breakdown uncertainty, the low, medium and high levels represent 10, 30 and 50 percent reduction of the maximum plant production capacity. The production capacity reduction is modeled by 8 breakdown scenarios: in scenario 1 no breakdown occurs, whereas in scenarios 2-8 a breakdown occurs in periods $1-4, \ldots, 22-24$. The probabilities are $50 \%$ for scenario 1 and $(50 / 7) \%$ for scenarios $2-8$. So there is a set of breakdown scenarios, $S_{B}$, with probabilities $\phi_{B}$ and cardinality equal to $8\left(\left|S_{B}\right|=8\right)$. Similarly, we define a set of equiprobable deviation price scenarios, $S_{P}$, with probability $\phi_{P}$ and cardinality $\left|S_{P}\right|$. For the deviation prices uncertainty, we consider three levels of uncertainty - low, medium, and high, that represent variations of 10,30 and 50 percent around the expected values. Each pair of breakdown scenario and penalty price scenario represents a general scenario $s$ with probability equal to $\phi_{s}=\phi_{B} * \phi_{P}$.

When deviation price uncertainty is considered, each scenario corresponds to a time series of devation prices for each time period of the scheduling horizon. The deviation price is assumed to be proportional to the day-ahead electricity price. Monte Carlo simulation was applied to generate 1000 scenarios. The large number of scenarios is required to accurately characterize the uncertainty, since the uncertain parameter (deviation price) can change over time. However the large number of scenarios may render the optimization problem computationally intractable. To reduce the number of scenarios and, at the same time, preserving the main features of the uncertainty, we applied the scenario reduction technique proposed in [7]. The reduction algorithms defines a probability distance to trade off scenario probabilities and distances of scenario values. Deletion will occur if scenarios are close or have small probabilities. In this work, we applied the forward reduction method implemented in the scenario reduction function SCENRED in GAMS [10]. We reduced the number of scenarios to $40\left(\left|S_{P}\right|=40\right)$. As an example, the deviation price profiles and the expected deviation price profile for the medium uncertainty level are shown in Figure 2. Future works will adopt more complicated price prediction models, e. g., ARIMA models. We refer the reader to [1] for 


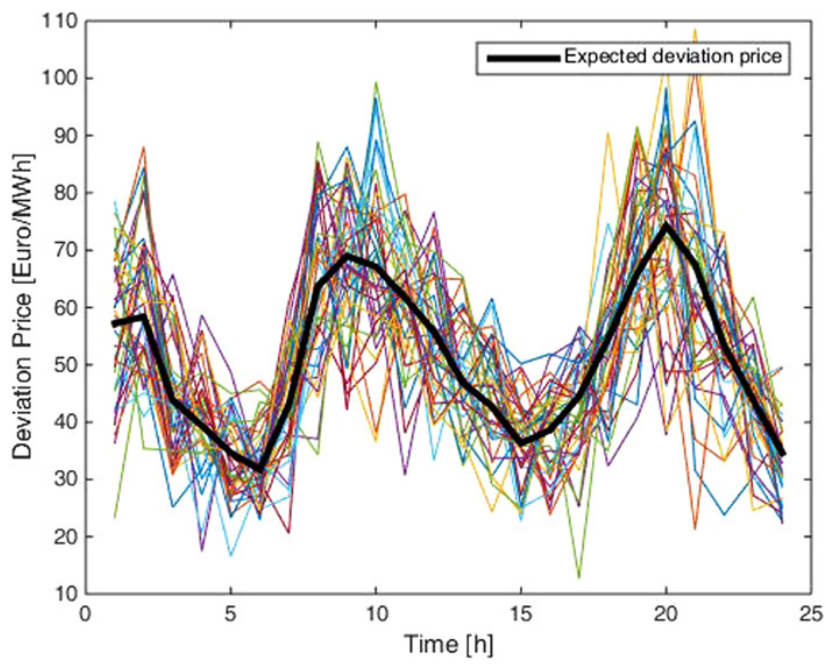

Figure 2: Random deviation price profiles associated with the medium uncertainty level.

a review of tools of price forecasting, which is not the focus of this work.

\subsection{MILP formulation}

To demonstrate the potential of the proposed approach, we apply it to a continuous production plant. The plant is the same as in [33]. In the proposed mathematical formulation, here-and-now decisions have no scenario subscript and wait-and-see decisions have the scenario subscript $s$. The indices, sets, parameters, and variables used in the MILP formulation are listed in the Nomenclature section.

\section{Nomenclature}

\section{Indices}

$i$
$t, t \prime$
$m, m \prime$
$s$
$j$

\section{Sets}

\section{$I$}

$T$

$M$

$S$ products

time periods

operating modes

scenarios

vertices of the plant operating region

products

time periods

operating modes

scenarios

\section{Parameters}

$v_{m j i s} \quad$ amount of product $i$ produced at vertex $j$ of mode $m$ in scenario $s[\mathrm{~kg}]$

$\theta_{m, m l, t} \quad$ minimum stay time in mode $m$ after switching from mode $m \prime$ [h]

$\delta_{m}$

$\gamma_{m i}$

$I V_{i t}^{\max }, I V_{i t}^{\min }$

constant electricity consumption in mode $m[\mathrm{kWh}]$

electricity consumption coefficient for production of product $i$ in mode $m$ $[\mathrm{kWh} / \mathrm{kg}]$

upper and lower bounds of the inventory level of product $i$ at time period $t[\mathrm{~kg}]$

$I V_{i}^{\text {initial }}, I V_{i}^{\text {final }}$

initial and final inventory level of product $i[\mathrm{~kg}]$

$y_{\text {m,initial }} \quad$ active operating mode at the beginning of the time horizon

$D_{i t}$

$E_{\text {max }}^{P P}$

demand of product $i$ at time period $t[\mathrm{~kg}]$

upper bound of the electricity generation [kWh]

$c^{g} \quad$ electricity generation cost [€/kWh]

$c^{s} \quad$ start-up cost of the electricity generation plant $[€]$

$k_{y} \quad$ reduction coefficient of electricity generation during start-up

$r_{\text {min }}, d_{\text {min }}$

$p_{t}^{\text {day-ahead }}$

minimum runtime and minimum downtime of the electricity generation plant $[\mathrm{h}]$ day-ahead electricity price for time period $t[€ / \mathrm{kWh}]$

$\phi_{s}$ $p_{s t}^{+}, p_{s t}^{-}$ probability of scenario $s$

deviation price for over- and underelectricity consumption at time period $t$ in scenario $s[€ / \mathrm{kWh}]$

purchasing price of product $i[\mathrm{~kg}]$

quantile for the definition of the risk measure $C V$

$\eta \quad$ scalar parameter to define objectives priorities

\section{Continuous variables}

$P D_{i t s}$

$\overline{P D}_{\text {mits }}$

$\lambda_{\text {mits }}$

$E U_{t s}$ amount of product $i$ produced at time $t$ in scenario $s$

amount of product $i$ produced at time $t$ in mode $m$ in scenario $s$

coefficients for vertex $j$ of the operating region in mode $m$ at time period $t$ in scenario $s$ total electricity consumption at time $t$ in scenario $s$ 


\begin{tabular}{|c|c|}
\hline$I V_{i t s}$ & $\begin{array}{l}\text { inventory levels of product } i \text { at time } t \text { in } \\
\text { scenario } s\end{array}$ \\
\hline$S L_{i t s}$ & $\begin{array}{l}\text { amount of product } i \text { sold at time } t \text { in sce- } \\
\text { nario } s\end{array}$ \\
\hline$P W_{i t s}$ & $\begin{array}{l}\text { amount of product } i \text { wasted at time } t \text { in } \\
\text { scenario } s\end{array}$ \\
\hline$P C_{i t s}$ & $\begin{array}{l}\text { amount of product } i \text { purchased at time } t \text { in } \\
\text { scenario } s\end{array}$ \\
\hline$E_{t s}^{P P}$ & $\begin{array}{l}\text { electricity generated at time } t \text { in scenario } \\
s\end{array}$ \\
\hline$y_{t}^{P P}$ & $\begin{array}{l}1 \text { if the electricity generation plant is } \\
\text { started-up at time } t \text { (pseudo-continuous } \\
\text { variable) }\end{array}$ \\
\hline$E S_{t}$ & $\begin{array}{l}\text { day-ahead electricity commitment at time } \\
t\end{array}$ \\
\hline$\delta e_{t s}^{+}, \delta e_{t s}^{-}$ & $\begin{array}{l}\text { electricity over-consumption and under- } \\
\text { consumption at time } t \text { in scenario } s\end{array}$ \\
\hline$z$ & total expected cost \\
\hline $\bar{z}$ & $\begin{array}{l}\text { weighted sum of the total expected cost } \\
\text { and the CVaR }\end{array}$ \\
\hline CVaR & conditional value at risk \\
\hline$\psi, \zeta_{s}$ & continuous variables to define $C V a R$ \\
\hline
\end{tabular}

\section{Binary variables}

$$
\begin{aligned}
& y_{m t} \\
& z_{m, m ı, t} \\
& x_{t}^{P P}
\end{aligned}
$$

1 if the operating mode $m$ is active in time period $t$

1 if transition from mode $m$ to mode $m ı$ occurs at time $t$

1 if the electricity generation plant is operating at time $t$

\subsubsection{Plant model}

The plant produces two products $i(P 1$ and $P 2)$ and it can operate in three different modes $m$ : off, startup, and on . The amount of product $i$ produced in time period $t$ of scenario $s$ is denoted by $P D_{i t s}$ and it is defined by Eq. (10) as the sum over all the operating modes of the variable $\overline{P D}_{\text {mits }}$ that denotes the amount of product $i$ produced in time period $t$ for mode $m$ of scenario $s$. For each mode, the operating conditions are expressed as a convex combination of the extreme points $v_{\text {mjis }}$ of the feasible region of operation (Eq. (11)). The vertices of the polyhedral feasible regions of each mode are listed in Table 1 and Figure 3 shows the polyhedral feasible region of mode on for the nominal case without equipment breakdown and for different breakdown levels. The binary variable $y_{m t}$ is 1 if mode $m$ is selected in time period $t$ of the horizon $T$. Eq. (12) forces
Table 1: Vertices associated with each operating mode of the plant in the nominal case with no breakdown.

\begin{tabular}{lrrr}
\hline Mode & Vertex & $\boldsymbol{P 1}[\mathbf{k g}]$ & $\boldsymbol{P 2}[\mathbf{k g}]$ \\
\hline Off & 1 & 0 & 0 \\
Startup & 1 & 5 & 5 \\
On & 1 & 10 & 10 \\
On & 2 & 50 & 10 \\
On & 3 & 30 & 40 \\
On & 4 & 70 & 40 \\
\hline
\end{tabular}

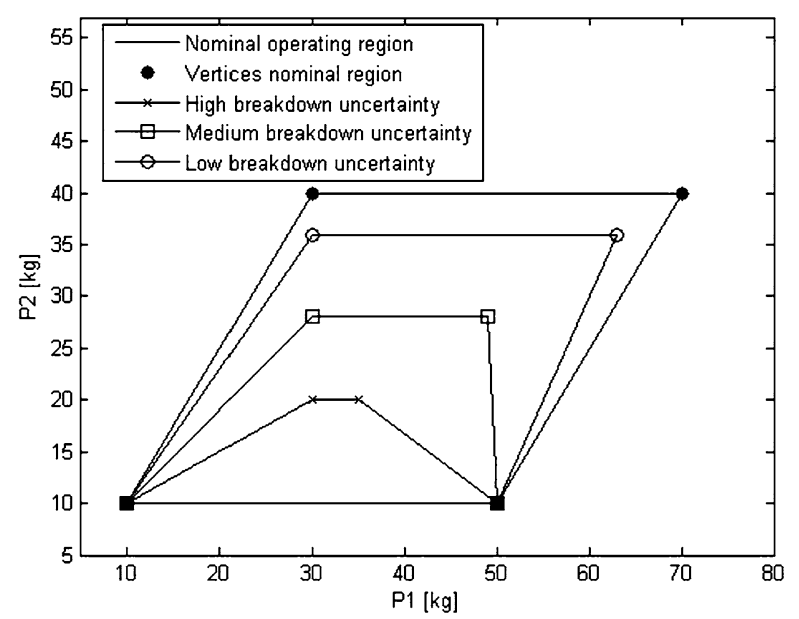

Figure 3: Polyhedral operating region of mode on with different uncertainty levels (the low, medium and high levels represent 10 , 30 and 50 percent reductions of the maximum plant production capacity).

the sum of the coefficients of the convex combination of the extreme points $v_{m j i s}$ to be equal to 1 if the related mode $m$ is active in time period $t$ or equal to 0 in case it is not active in time period $t$. Eq. (13) ensures that only one mode is active for each time period $t$. The possible mode transitions are off to startup, startup to on, and on to off and they can happen only after fixed periods of time, $\theta_{m m l}$, that have been spent in the modes (off: $8 \mathrm{~h}$, startup: $2 \mathrm{~h}$, on: $6 \mathrm{~h}$ ). The binary variable $z_{m m, t}$ takes value of 1 if and only if the plant switches from mode $m$ to mode $m \prime$ in time period $t$. This is enforced by Eq. (14). Eq. (15) restricts the plant to remain in a certain mode $m$ ' for an amount of time equal to $\theta_{m m}$ after the transition from mode $m$. Eq. (16) defines the total amount of electricity consumed in time period $t$ and scenario $s, E U_{t s}$, as the sum over the operating modes of a fixed term $\delta_{m}$ (if the mode $m$ is active) and a linear function of the hourly production $\overline{P D}_{\text {mits }}$. The electricity consumption coefficients for each mode of operation are shown in Table 2.

$$
P D_{i t s}=\sum_{m} \overline{P D}_{m i t s} \forall i, t \in T, s
$$


Table 2: Electricity consumption coefficients for each operating mode.

\begin{tabular}{lrrr}
\hline Mode & $\delta_{m}[\mathrm{kWh}]$ & $\gamma_{P 1}[\mathrm{kWh} / \mathrm{kg}]$ & $\gamma_{P 2}[\mathrm{kWh} / \mathrm{kg}]$ \\
\hline Off & 0 & 0 & 0 \\
Startup & 500 & 0 & 0 \\
On & 800 & 20 & 30 \\
\hline
\end{tabular}

$$
\begin{aligned}
& \overline{P D}_{m i t s}=\sum_{j} \lambda_{m j t s} * v_{m j i s} \quad \forall i, t \in T, s, m \in M \\
& \sum_{j} \lambda_{m j t s}=y_{m t} \quad \forall t \in T, s \\
& \sum_{m} y_{m t}=1 \quad \forall t \in T \\
& \sum_{m \prime} z_{m \prime m, t-1}-\sum_{m \prime} z_{m m ! t-1}=y_{m t}-y_{m, t-1} \quad \forall m, t \in T \\
& \sum_{k=1}^{\theta_{m m \prime}} z_{m m l, t-k} \leq y_{m \prime t} \quad \forall(m, m \prime) \in M, t \in T \\
& E U_{t s}=\sum_{m}\left(\delta_{m} * y_{m t}+\sum_{i} y_{m i} * \overline{P D}_{m i t s}\right) \\
& \forall t \in T, s
\end{aligned}
$$

Note the binary variables $y_{m t}, z_{m m ! t}$ are here-and-now decisions, while the production levels $\overline{P D}_{\text {mits }}, P D_{\text {its }}$ and the energy consumption $E U_{t s}$ are wait-and-see decisions that can be optimally adapted to information that becomes available.

\subsubsection{Inventory balance}

The products that are produced by the plant can be stored and Eq. (17) defines for each scenario $s$ the inventory level $I V_{\text {its }}$ of product $i$ at time $t$ as the sum of the inventory level of product $i$ at time period $t-1$ and the production level of product $i$ at time $t, P D_{i t s}$, minus the amount of product $i$ sold, $S L_{i t s}$, and the amount of product $i$ wasted, $P W_{i t s}$, at time period $t$. Note that all these variables are wait-and-see variables and they are defined for each scenario s. Eq. (18) sets upper and lower bounds of the inventory levels and Eq. (19) ensures that the demand of product $i$, defined as the sum of the amount of product $i$ sold, $S L_{i t s}$, and the amount of product $i$ purchased from other sources, $P C_{i t s}$, is satisfied. Lower and upper bounds for the inventory levels and the product demand values are listed in Table 3.

$$
\begin{aligned}
& I V_{i t s}=I V_{i, t-1, s}+P D_{i t s}-S L_{i t s}-P W_{i t s} \quad \forall i, t \in T, s \\
& I V_{i t}^{\min } \leq I V_{i t s} \leq I V_{i t}^{\max } \quad \forall i, t \in T, s \\
& S L_{i t s}+P C_{i t s}=D_{i t} \quad \forall i, t \in T, s
\end{aligned}
$$

Table 3: Upper bounds and lower bounds of the inventory levels.

\begin{tabular}{rrrr}
\hline & $\boldsymbol{I V}_{\min } / \boldsymbol{I V}_{\boldsymbol{m a x}}[\mathrm{kg}]$ & $\boldsymbol{I} \boldsymbol{V}_{\text {init }} / \boldsymbol{I} \boldsymbol{V}_{\text {fin }}[\mathrm{kg}]$ & $\boldsymbol{D}_{\boldsymbol{t}}$ \\
\hline P1 & $600 / 6000$ & $1000 / 1000$ & 60 \\
P2 & $300 / 3000$ & $500 / 500$ & 35 \\
\hline
\end{tabular}

\subsubsection{Initial and final conditions}

Eqs. (20)-(21) provide the initial condition of the plant in terms of inventory levels and active operating mode. Eq. (22) imposes a terminal constraint for the inventory levels of product $i$. Eq. (23) provides information regarding the mode switching before the time of horizon of interest.

$$
\begin{aligned}
I V_{i, 0, s} & =I V_{i}^{\text {initial }} \forall i, s \\
y_{m, 0} & =y_{m, \text { initial }} \quad \forall m \\
I V_{i, t^{\text {final }, s}} & \geq I V_{i}^{\text {final }} \quad \forall i, s \\
z_{m m, t} & =z_{m m / t}^{\text {initial }} \quad \forall(m, m \prime) \in M,-\theta^{\max }+1 \leq t \leq-1
\end{aligned}
$$

\subsubsection{On-site generation}

The production plant is equipped with a power plant for the on-site generation of electric power. The binary variable $x_{t}^{P P}$ takes the value of 1 at time period $t$ if and only if the power plant generates electricity at time period $t$. An auxiliary pseudo-continuous variable $y_{t}^{P P}$ indicates startup of generation: the pseudo-continuous variable $y_{t}^{P P}$ is equal to 1 at time period $t$ if the power plant is started up at time period $t$. This is enforced by Eqs. (24-25). Eqs. (26-27) define a minimum runtime $r$ min and a minimum downtime $d \mathrm{~min}$. The amount of electricity generated by the power plant at time period $t$ in scenario $s$ is defined by the continuous variable $E_{t s}^{P P}$. Eq. (28) defines the upper bound of the electricity generation levels and reduces the electricity generation levels by a factor $k_{y}$ for time period $t$ when start-up occurs. Eq. (29) defines the total electricity generation $\operatorname{cost} c_{t s}^{P P}$ at time period $t$ in scenario $s$ as a linear function of the generation level at time period $t$ in scenario $s$, $E_{t s}^{P P}$, with an additional constant $\cos t c^{s}$ for the time period $t$ when the power plant is started up. The parameters related to the electricity generation are listed in Table 4. The efficiency of a conventional power plant usually depends nonlinearly on the generation level. However, to avoid a mixed-integer nonlinear formulation, it is common practice to linearize the power plant efficiency within the plant operating region.

$$
x_{t}^{P P}-x_{t-1}^{P P} \leq y_{t}^{P P} \leq x_{t}^{P P} \quad \forall t \in T
$$


Table 4: On-site generation parameters.

\begin{tabular}{lr}
\hline$c^{g}[€ / \mathrm{MWh}]$ & 50 \\
$r_{\min }[\mathrm{h}]$ & 2 \\
$d_{\min }[\mathrm{h}]$ & 2 \\
$c_{\text {startup }}[€]$ & 50 \\
$k_{y}[\%]$ & 20 \\
\hline
\end{tabular}

$$
\begin{aligned}
0 & \leq y_{t}^{P P} \leq 1-x_{t-1}^{P P} \quad \forall t \in T \\
\sum_{t \prime=t}^{t+r_{\text {min }}-1} x_{t \prime}^{P P} & \geq r_{\text {min }} *\left(x_{t}^{P P}-x_{t-1}^{P P}\right) \quad \forall t<|T|-r_{\text {min }} \\
\sum_{t \prime=t}^{t+d_{\text {min }}-1} x_{t \prime}^{P P} & \leq d_{\text {min }} *\left(1+x_{t}^{P P}-x_{t-1}^{P P}\right) \quad \forall t<|T|-d_{\text {min }} \\
E_{t s}^{P P} & \leq E_{\text {max }}^{P P} * x_{t}^{P P}-k_{y} * E_{\text {max }}^{P P} * y_{t}^{P P} \quad \forall t \in T, s \\
c_{t s}^{P P} & =c^{g} * E_{t s}^{P P}+c^{s} * y_{t}^{P P} \quad \forall t \in T, s
\end{aligned}
$$

\subsubsection{Day-ahead electricity commitment}

The plant can purchase electricity from the day-ahead market. The day-ahead electricity commitment at each time period $t$ is defined by the continuous variable $E S_{t}$. Eq. (30)-(31)-(32) define for each time period $t$ the dayahead electricity commitment $E S_{t}$, the electricity overconsumption $\delta e_{t s}^{+}$and the electricity under-consumptions $\delta e_{t s}^{-}$. Electricity over-consumption and electricity underconsumption are the load deviations respect to the dayahead electricity commitment and therefore defined as the difference between the actual consumption of electric power, $E U_{t s}$, the day-ahead commitment $E S_{t}$ and the amount of electricity generated on-site $E_{t s}^{P P}$. No upper and lower bounds are set for the over-consumption and underconsumption of electric power. Since the plant has to commit itself to the amount of energy to be purchased for a period of 24 hours one day before the actual electricity consumption is realized, electricity commitment decisions are first-stage variables and load deviations are second stage variables.

$$
\begin{aligned}
E U_{t s}-E S_{t}-E_{t s}^{P P} & =\delta e_{t s}^{+}-\delta e_{t s}^{-} \quad \forall t \in T, s \\
\delta e_{t s}^{+} & \geq 0 \quad \forall t \in T, s \\
\delta e_{t s}^{-} & \geq 0 \quad \forall t \in T, s
\end{aligned}
$$

\subsubsection{Objective function}

The expression of the objective function differs for the case of risk-neutral and risk-averse optimization. In the case of risk-neutral optimization the model minimizes the total expected operating cost; in the case of risk-averse optimization the objective function is the weighted sum of the total expected operating cost and the risk measure adopted, the CVaR in our formulation.

\subsubsection{Risk-neutral optimization}

The model minimizes the total expected operating cost, $z$, defined by Eq. (33) as the sum of the first-stage cost and the expected second-stage cost. The first-stage cost is the cost of purchasing electricity from the day-ahead market at the hourly-varying day-ahead electricity price. For each scenario $s$ the second-stage cost consists of the sum of the cost of load deviation, the cost of on-site electricity generation and the cost of purchasing of products on the market.

$$
\begin{aligned}
z=\sum_{t}\left(p_{t}^{\text {day-ahead }}\right. & * E S_{t}+\sum_{s} \phi_{s} *\left(p_{s t}^{+} * \delta e_{t s}^{+}+\right. \\
+ & \left.\left.p_{s t}^{-} * \delta e_{t s}^{-}+c_{t s}^{P P}+\sum_{i} p_{i} * P C_{i t s}\right)\right)
\end{aligned}
$$

where $p_{t}^{\text {day-ahead }}, p_{s t}^{+}, p_{s t}^{-}, p_{i}$ represent the day-ahead electricity price, the penalty cost for over consumption and under consumption and the product purchasing prices; $\phi_{s}$ denotes the probability of scenario $s$.

\subsubsection{Risk-averse optimization}

For risk-averse optimization, the model minimizes the weighted sum of the total expected cost and the CVaR, $\bar{z}$, defined by Eq. (37). The scalar parameter $\eta \in[0, \ldots, 1]$ allows the decision maker to prioritize the two objectives. According to [25], the CVaR for the quantile $\alpha$ can be defined by the linear constraints in Eqs. (34)-(35)-(36).

$$
\begin{aligned}
& C V a R=\min _{\psi, \zeta_{s}}\left\{\psi+(1-\alpha)^{-1} \sum_{s} \phi_{s} * \zeta_{s}\right\} \\
& \sum_{t}\left(p_{t}^{\text {day-ahead }} * E S_{t}+p_{s t}^{+} * \delta e_{t s}^{+}+p_{s t}^{-} * \delta e_{t s}^{-}+c_{t s}^{P P}\right. \\
&\left.+\sum_{i} p_{i} * P C_{i t s}\right)-\psi \leq \zeta_{s} \forall s \\
& \zeta_{s} \geq 0 \\
& \bar{z}=\eta * z+(1-\eta) * C V a R
\end{aligned}
$$

where $\psi, \zeta_{s}$ are continuous variables and $\phi_{s}$ denotes the probability of scenario $s$. Eqs. (35)-(36) impose that for each scenario $s$ in which the total cost is greater than $\psi$, 


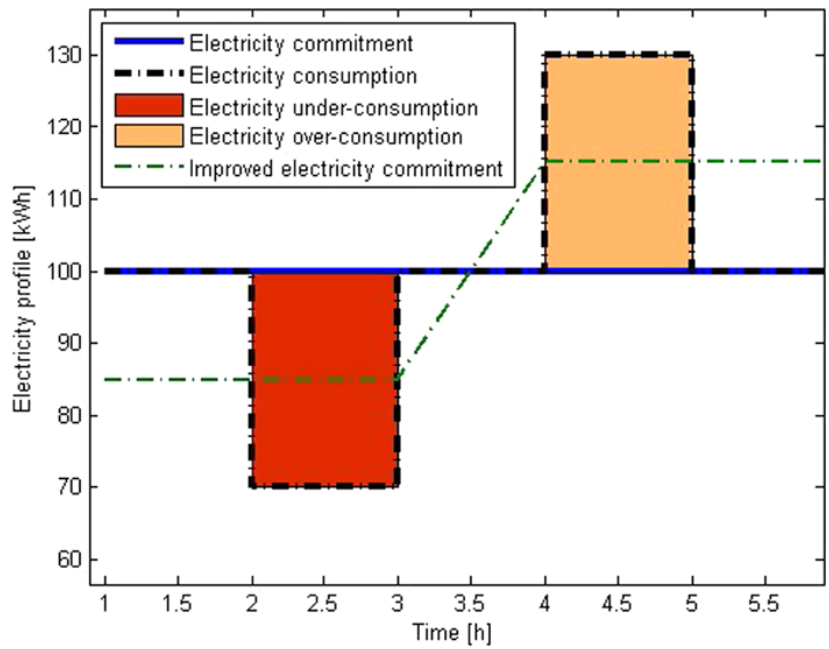

Figure 4: Illustrative example.

the variable $\zeta_{s}$ takes the value of the difference between the total cost and $\psi$; otherwise $\zeta_{s}$ takes the value of zero. Note that the variable $\psi$ takes the value of $V a R_{\alpha}$ when the CVaR is minimized.

\section{Discussion}

Before presenting the results of the proposed case-study, it is worth to analyze the simplified situation depicted in Figure 4. Here a constant electricity commitment of $100 \mathrm{kWh}$ for 6 time periods is shown by a blue line. The actual electricity consumption occurring after the realization of the equipment breakdown is represented by a dashed black line. The difference between the electricity commitment and the electricity consumption defines the load deviations. In particular the red block shows the underconsumption of electric power due to the reduction of the plant production capacity and the orange block shows the over-consumption of electric power which is necessary to restore the inventory level or to fulfill demand satisfaction constraints. The green dashed line shows how the load deviation costs can be reduced. It represents an electricity commitment profile that decreases the electricity deviations in the case the decision maker was aware of the breakdown realization: the electricity commitment (and therefore the production level) is lowered to reduce the under-consumption and after the breakdown realization it is increased to reduce the over-consumption. Clearly, the larger the area of the red block that is cut off by the electricity commitment profile, the larger is the reduction of the electricity deviation cost. The stochastic approach follows this idea by defining an optimal electricity commit-

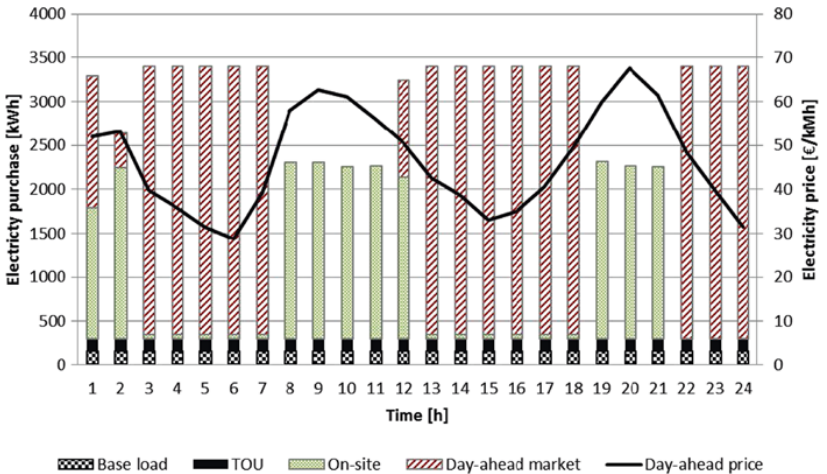

Figure 5: Electricity purchase profiles obtained from solving the deterministic model with medium uncertainty level. Black line: dayahead price of electric power.

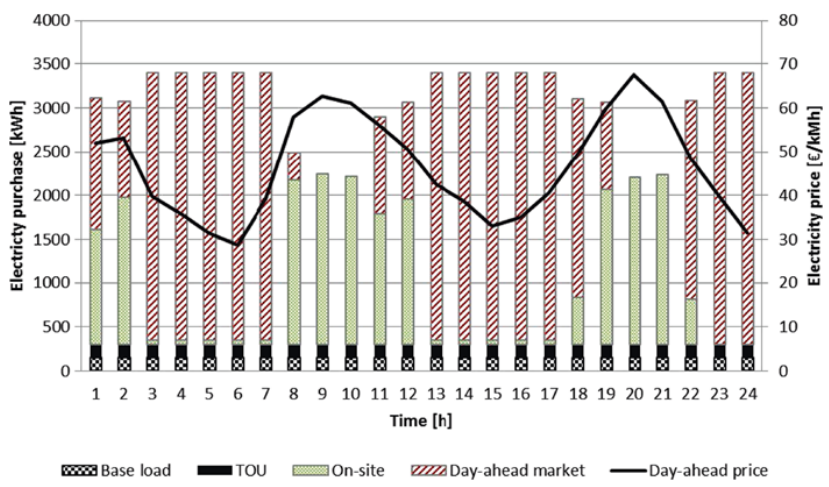

Figure 6: Electricity purchase profiles obtained solving the riskneutral stochastic model with medium uncertainty level.

ment (first-stage variable) under the assumption that load deviations are optimally adapted to the realization of the uncertainty and that the realization of the uncertainty is only known probabilistically.

The same explanation is valid for the results of the case-study shown in Figures 5-6. Here the electricity purchase profiles and the on-site electricity generation profiles that were obtained solving the deterministic model and the risk-neutral stochastic model with medium uncertainty level are depicted. TOU and base load profiles represent the amount of energy purchased from the power contracts. TOU and base load profiles are supposed to be given and therefore not optimized since contract related decisions have to be made before the time horizon of interest (1 week before for the TOU contract and 1 year before for the base load contract). Note that start-up/shut-down decision regarding the power plant are first-stage decisions, but the hourly electricity generation levels are second-stage decisions. The generation profiles shown are the expected values computed over all the scenarios. 


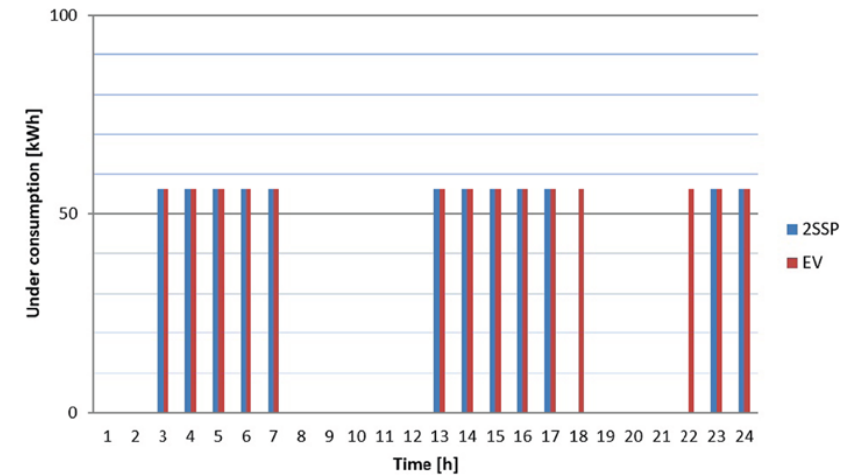

Figure 7: Expected under-consumption obtained solving the deterministic model and the risk-neutral stochastic model with medium uncertainty level.

The comparison between the stochastic and deterministic solutions shows the impact on the day-head electricity commitment and on the on-site generation strategy (first-stage variables) of accounting for the other scenarios besides the expected one. In the deterministic solution (Figure 5) the electricity is purchased from the day-ahead market exclusively according to the day-ahead electricity price and the on-site generation plant produces electricity when the on-site generation cost is lower than the electricity price: during off-peak periods (e. g., 3-6 h) the amount of electricity purchased (red bars) is increased in order to increase the production level and to reduce the electricity purchase during periods of peak prices (at 9-10 $\mathrm{h}$ and 20$21 \mathrm{~h}$ ). During these price-peak intervals the power plant is started up to provide electricity to the production plant (green bars). The energy consumption and therefore the production are shifted to time intervals when the electricity price is lower. The stochastic solution defines the electricity commitment not only according to the electricity price and the on-site generation cost but also accounting for possible breakdown scenarios. The stochastic solution (Figure 6) reduces the electricity over-consumption by increasing the amount of electricity purchased during the time intervals when production capacity is still available and the deviation price is convenient. On the other hand, the stochastic solution minimizes the under-consumption due to the equipment breakdown reducing the energy commitment and increasing the on-site electricity generation (e. g., at 18 and $22 \mathrm{~h}$ ). In fact, in the stochastic solution the power plant operates longer in order to accommodate for the breakdown scenarios. Figures 7-8 show the expected under-consumption and the expected overconsumption obtained by solving the deterministic model and the risk-neutral stochastic model. Note that the underconsumption and the over-consumption are second-stage variables and therefore different for each scenario.

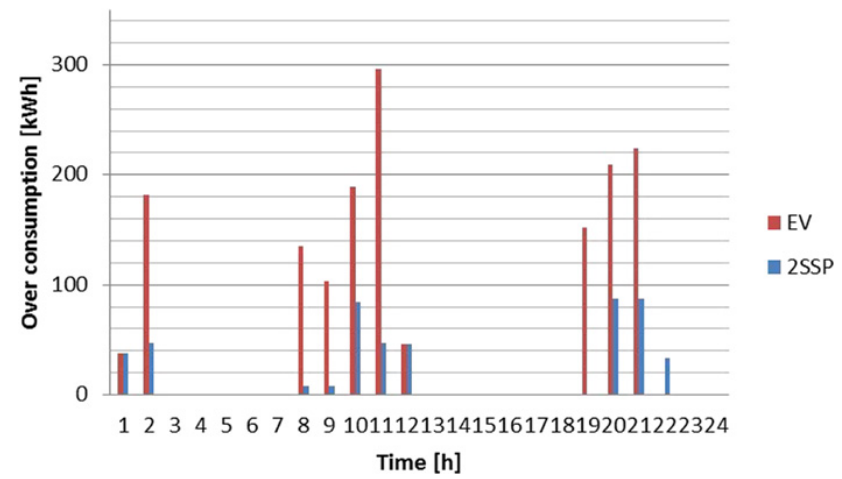

Figure 8: Expected over-consumption obtained solving the deterministic model and the risk-neutral stochastic model with medium uncertainty level.

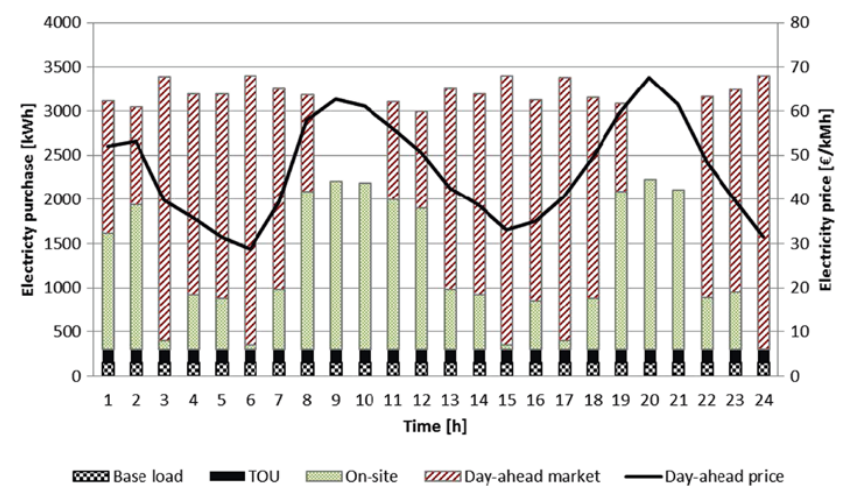

Figure 9: Electricity purchase profiles obtained solving the riskaverse stochastic model with medium uncertainty level.

Figure 9 shows the electricity purchase profiles and the on-site electricity generation profiles obtained by solving the risk-averse stochastic model with medium uncertainty level. The risk-averse solution, compared to the riskneutral solution (Figure 6), increases the on-site electricity generation level, even when the electricity price decreases (e. g., 4-5 h), in order to avoid high cost scenarios.

\section{Results}

To measure the improvement that can be achieved by solving the stochastic model instead of the deterministic counterpart, we compute the value of the stochastic solution (VSS). Table 5 shows the VSS for different levels of uncertainty obtained when solving the risk-neutral and the riskaverse optimization. Here, $\left|S_{B}\right|$ and $\left|S_{P}\right|$ denote the number of breakdown scenarios and deviation price scenarios. As described in Section 4.1, the number of breakdown scenar- 
Table 5: Value of the stochastic solution for different levels of uncertainty.

\begin{tabular}{|c|c|c|c|c|c|c|c|}
\hline$\left|S_{B}\right|$ & $\sigma_{B}$ & $\left|S_{P}\right|$ & $\sigma_{P}$ & VSS $[€]$ & $\overline{V S S}[\%]$ & VSS [€] (risk-averse) & $\overline{V S S}$ [\%] (risk-averse) \\
\hline & & 40 & Low & 13 & 0.4 & 35 & 1.0 \\
\hline \multirow[t]{2}{*}{8} & Low & 40 & Medium & 23 & 0.8 & 76 & 2.3 \\
\hline & & 40 & High & 30 & 0.9 & 98 & 2.7 \\
\hline \multirow{3}{*}{8} & & 40 & Low & 38 & 1.1 & 131 & 3.6 \\
\hline & Medium & 40 & Medium & 93 & 2.7 & 278 & 7.5 \\
\hline & & 40 & High & 125 & 3.5 & 351 & 9.1 \\
\hline \multirow{3}{*}{8} & & 40 & Low & 79 & 2.2 & 272 & 7.13 \\
\hline & High & 40 & Medium & 210 & 5.7 & 570 & 13.8 \\
\hline & & 40 & High & 265 & 7.0 & 671 & 15.5 \\
\hline
\end{tabular}

ios is equal to $8\left(\left|S_{B}\right|=8\right)$ and the number of penalty price scenarios is equal to $40\left(\left|S_{P}\right|=40\right)$. Note that since both the uncertainty sources are considered, the total number of scenarios is equal to $\left|S_{P}\right| *\left|S_{B}\right|=320$.

It can be seen from Table 5 that the VSS can be quite significant and that it grows with the level of uncertainty. There is benefit from accounting for uncertainty for a continuous plant equipped with a power plant. In the case of low breakdown rate, the plant is able to compensate the reduction of production capacity with the product inventory. Therefore the VSS is small. For the case of high breakdown level scenarios the plant might have to purchase the products from other sources in order to fulfill the demand satisfaction constraints. Note that because of the possibility to purchase products from other sources (and because of the absence of upper bounds for the electricity deviation variables), the problem is a stochastic problem with complete recourse, since for any choice of the first-stage variables (even for no electricity commitment) the second-stage constraints can always be satisfied.

Table 5 compares the VSS obtained considering the risk-neutral objective function and the risk-averse objective function. We choose $\eta$ equal to 0.5 (Eq. (37)) to assign equal weights to the total expected profit and the CVaR. The confidence level $\alpha$ at which the CVaR is defined is equal to 0.9 (Eq. (34)). It is evident that accounting for risk can lead to a significant added value.

Each model has approximately 255.000 continuous variables, 233 binary variables, and 163.000 constraints. All models were solved to zero integrality gap in less than $60 \mathrm{~s}$ on an Intel(R) Core(TM) i7-4790 machine at $3.60 \mathrm{GHz}$ with eight processors and 16 GB RAM. All models were implemented in GAMS 24.7.4 (GAMS Development Corporation, 2015), and the MILPs were solved applying the commercial solver CPLEX 12.6.3.0.

\section{Conclusion}

This work addresses the integrated day-ahead electricity commitment and production scheduling for powerintensive processes. A two-stage stochastic programming approach has been proposed to face the uncertainty in the decision process since the electricity consumers have to commit to the amount of energy they are going to purchase from the power grid before the actual electricity demand is known. A MILP formulation has been proposed to model a continuous production plant and an on-site power plant. The application of the proposed approach shows the benefit from accounting for uncertainty to solve the integrated day-ahead electricity commitment and production scheduling problems. Different sources of uncertainty and different levels of uncertainty have been analyzed. Future works will apply the proposed approach to batch processes what renders the optimization problem much harder since the solution of a batch scheduling problem is needed to evaluate the second-stage cost for each scenario. To this aim the design of advanced decomposition strategies able to exploit the problem structure will be investigated.

Funding: Financial support is gratefully acknowledged from the Marie Skłodowska Curie Horizon 2020 EID-ITN project "PROcess NeTwork Optimization for efficient and sustainable operation of Europe's process industries taking machinery condition and process performance into account - PRONTO”, Grant agreement No 675215.

\section{References}

1. S. Aggarwal, L. Saini and A. Kumar, Electricity price forecasting in deregulated markets: A review and evaluation, International Journal of Electrical Power \& Energy Systems 31 (2009), 13-22.

2. J. R. Birge and F. Louveaux, Multistage Stochastic Programs, Introduction to Stochastic Programming (1997), 233-252. 
3. J. R. Birge and F. Louveaux, Two-Stage Linear Recourse Problems, 1997.

4. C. C. Carøe and R. Schultz, Dual decomposition in stochastic integer programming, Operations Research Letters 24 (1999), 37-45.

5. P. M. Castro, I. Harjunkoski and I. E. Grossmann, Optimal scheduling of continuous plants with energy constraints, Comput Chem Eng 35(2) (2011), 372-387.

6. P. M. Castro, L. Sun and I. Harjunkoski, Resource - Task Network Formulations for Industrial Demand Side Management of a Steel Plant, Industrial and Engineering Chemistry Research 52(36) (2013), 13046-13058.

7. J. Dupacova, N. Growe-Kuska and W. Romisch, Scenario reduction in stochastic programming: An approach using probability metrics, Math. Program. Ser. A (2003), 493-511.

8. EEG, Renewable Energy Act, EEG 2009, in: Federal Law Gazette, Federal Ministry of Justice, Bonn; October 49.part I (2008).

9. S. Engell, Uncertainty, decomposition and feedback in batch production scheduling, in: 19th European Symposium on Computer Aided Process Engineering - ESCAPE19 (2009), 43-62.

10. GAMS Development Corporation. GAMS version 24.4.1, 2015.

11. A. M. Geoffrion, Generalized benders decomposition, Journal of Optimization Theory and Applications 10 (1972), 237-260.

12. M. Guignard, Lagrangean relaxation, Top 11 (2003), 151-200.

13. H. Hadera, I. Harjunkoski, G. Sand, I. E. Grossmann and S. Engell, Optimization of steel production scheduling with complex time - sensitive electricity cost, Computers and Chemical Engineering 76 (2015), 117-136.

14. R. R. Iyer and I. E. Grossmann, A bilevel decomposition algorithm for long-range planning of process networks, Industrial and Engineering Chemistry Research 37 (1998), 474-481.

15. K. Kim and V. M. Zavala, Algorithmic innovations and software for the dual decomposition method applied to stochastic mixed-integer programs, Optimization Online (2015).

16. S. Kuçukyavuz and S. Sen, An introduction to two-stage stochastic mixed-integer programming, Leading Developments from INFORMS Communities 11 (2017), 1-27.

17. G. Laporte and F. V. Louveaux, The integer l-shaped method for stochastic integer programs with complete recourse, Operations Research Letters 13 (1993), 133-142.

18. E. Leo and S. Engell, Multi-stage integrated electricity procurement and production scheduling, in: International Symposium on Process Systems Engineering - PSE 2018, July 1-5 2018.

19. E. Leo and S. Engell, A two-stage stochastic programming approach to integrated day-ahead electricity commitment and production scheduling, in: Proceedings of the 28th European Symposium on Computer Aided Process Engineering, June 10-13 2018.

20. L. Merkert, I. Harjunkoski, A. Isaksson, S. Saynevirta, A. Saarela and G. Sand, Scheduling and energy - industrial challenges and opportunities, Comput. Chem. Eng. 72 (2014), 183-198.

21. S. Mitra, I. E. Grossmann, J. M. Pinto and N. Arora, Optimal scheduling of industrial combined heat and power plants under time-sensitive electricity prices, Energy 54 (2013), 194-211.
22. K. Nolde and M. Morari, Electrical load tracking scheduling of a steel plant, Comput. Chem. Eng. 34(11) (2010), 1899-1903.

23. M. Paulus and F. Borggrefe, The potential of demand - side management in energy - intensive industries for electricity markets in Germany, Appl. Energy 88 (2011), 432.

24. R. T. Rockafellar, Coherent Approaches to Risk in Optimization Under Uncertainty, INFORMS Tutorials in Operations Research (2015), 38-61.

25. R. T. Rockafellar and S. Uryasev, Optimization of conditional value-at-risk, J Risk 2 (2000), 21-42.

26. R. T. Rockafellar and R. J.-B. Wets, Scenarios and policy aggregation in optimization under uncertainty, Mathematics of Operations Research 16 (2006), 203-223.

27. S. Sen and H. D. Sherali, Decomposition with branch-and-cut approaches for two-stage stochastic mixed-integer programming, Mathematical Programming 106 (2006), 203-223.

28. P. Siano, Demand response and smart grids - A survey, Renewable and Sustainable Energy Reviews 30 (2014), 461-478.

29. S. Uryasev, Conditional Value-at-Risk: Optimization Algorithms and Applications, Financial Engineering news 14 (2000).

30. S. S. Uryasev, Value-at-Risk vs. Conditional Value-at-Risk in Risk Management and Optimization, INFORMS Tutorials in Operations Research (2017).

31. Y. Qi and S. Sen, The ancestral benders' cutting plane algorithm with multi-term disjunctions for mixed-integer recourse decisions in stochastic programming, Mathematical Programming 161 (2006), 193-235.

32. Q. Zhang and I. E. Grossmann, Planning and scheduling for industrial demand side management: advances and challenges, Alternative energy sources and technologies: process design and operation (2015).

33. Q. Zhang, L. Jochen, L. Cremer, I. E. Grossmann, A. Sundaramoorthy and J. M. Pinto, Risk-based integrated production scheduling and electricity procurement for continuous power - intensive processes, Computers and Chemical Engineering 86 (2016), 90-105.

\section{Bionotes}

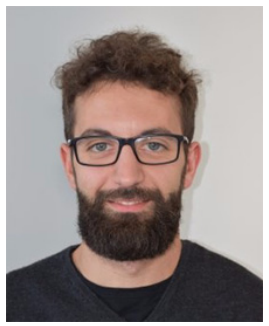

\section{Egidio Leo}

Process Dynamics and Operations Group, Department of Biochemical and Chemical Engineering, Technische Universität Dortmund, Emil-Figge-Str.70, 44221 Dortmund, Germany egidio.leo@tu-dortmund.de

Egidio Leo, M.Sc. (born 1988) received his M.Sc. degree in mechanical engineering - specialisation system dynamics, at Politecnico di Milano (Italy) in 2014. After working as a junior researcher at the National Italian Research Council, he joined the Process Dynamics and Operations Group at TU Dortmund University as a Marie Curie Early Stage Research Fellow, where he is working on his $\mathrm{PhD}$ in the field of stochastic integer programming. 


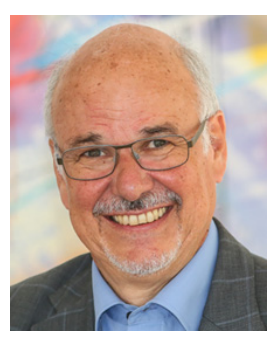

\section{Sebastian Engell}

Process Dynamics and Operations Group, Department of Biochemical and Chemical Engineering, Technische Universität Dortmund, Emil-Figge-Str.70, 44221 Dortmund, Germany

sebastian.engell@tu-dortmund.de

Prof. Dr.-Ing. Sebastian Engell (born 1954) received his Dipl.-Ing. degree in electrical engineering from the Ruhr-Universität Bochum, Germany, in 1978, and his Dr.-Ing. degree from the Universität Duisburg, Germany, in 1981. In 1990, he was appointed to his present position as a Full Professor of Process Dynamics and Operations with the Department of Biochemical and Chemical Engineering at the Technical University of Dortmund. His areas of research are robust optimizing control, in particular of chemical and biochemical processes, dynamic modeling, coordination in systems of systems, and planning and scheduling under uncertainty. He is recipient of an ERC Advanced Investigator Grant and Fellow of IFAC. 